\title{
REDESCRIÇÃO DE DUAS ESPÉCIES NEOTROPICAIS DE EMPIDIDAE (DIPTERA) DESCRITAS POR CURRAN E REVALIDAÇÃO DO GÊNERO PORPHYROCHROA
}

\author{
José A. Rafael ${ }^{1,2}$
}

\begin{abstract}
REDESCRIPTION OF TWO NEOTROPICAL SPECIES OF EMPIDIDAE (DIPTERA) DESCRIBED BY CURRAN AND REVALIDATION OF THE GENUS PORPHYROCHROA. The two species described in the genus Axelempis Curran, 1931, junior synonym of Macrostomus Wiedemann, 1817, Axelempis fulvithorax Curran, 1931 and A. fasciventris Curran, 1931 are redescribed from the types. The first one remains in the genus Macrostomus and the second one is transferred to Porphyrochroa Melander, 1928, here revalidated.
\end{abstract}

KEYWORDS. Diptera, Empididae, Macrostomus, Porphyrochroa, Taxonomy.

\section{INTRODUÇÃO}

CURRAn (1931) propôs o gênero Axelempis incluindo duas espécies, A. fulvithorax Curran, 1931 espécie-tipo e A. fasciventris Curran, 1931. Sмітн (1961) considerou Axelempis sinônimo junior de Macrostomus Wiedemann, 1817 baseando-se na forma da antena e na estrutura básica da terminália, semelhantes à espécie-tipo de Macrostomus, M. ferrugineus (Fabricius, 1805). A sinonímia está correta porque a espécie-tipo de Axelempis pertence ao gênero Macrostomus. No entanto, A. fasciventris, também transferida para Macrostomus, não apresenta as mesmas características da espécie anterior e está sendo transferida aqui ao gênero Porphyrochroa Melander, 1928, aqui revalidado.

1. Instituto Nacional de Pesquisas da Amazonia (INPA); Caixa Postal, 478; 69011-970, Manaus, Amazonas, Brasil (jarafael@inpa.gov.br).

2. Bolsista do CNPq. 
Os tipos das espécies redescritas e as espécies-tipo de Porphyrochroa e Macrostomus pertencem à coleção do American Museum of Natural History (AMNH), Nova Iorque; National Museum of Natural History (NMNH), Washington e Zoologisk Museum (ZM), Copenhagen. Uma das asas foi desprendida e montada em microlâmina com bálsamo do Canadá e a terminália, depois de dissecada e examinada, foi acondicionada em microtubo com glicerina. A terminologia adotada é a de McAlpine (1981), exceto para a terminália masculina que segue Cumming et al. (1995).

\section{Macrostomus fulvithorax (Curran, 1931)}

(Figs. 1-7, 10, 11)

Axelempis fulvithorax CURRAN, 1931:10.

Macrostomus fulvithorax; Sмітн, 1961:54, fig. 7; 1967:30 (cat.).

Diagnose. Tórax amarelo; abdômen castanho-avermelhado; pernas predominantemente amarelas; asa com enfuscação nas margens costal e distal; cerdas longas na tíbia e no primeiro tarsômero posterior do macho e cerdas peniformes na tíbia posterior da fêmea; primeiro tarsômero posterior da fêmea levemente intumescido.

Holótipo . Corpo $4 \mathrm{~mm}$ (do artigo original), asa 3,9 mm de comprimento. Cabeça: olhos dicópticos, castanhos; facetas inferiores levemente maiores. Fronte preta, brilhante, levemente mais estreita do que o ocelo anterior. Face com pruína cinza, da mesma largura e altura da fronte. Cerdas ocelares subparalelas, proclinadas; pós-ocelares ausentes. Pós-crânio largo, castanho-escuro a preto, brilhante na margem do olho e castanho posteriormente, com leve pruinosidade cinza, exceto por uma mancha dorsal, brilhante; cerdas pós-oculares unisseriadas, curtas, distintas; cerda vertical interna pouco maior do que a ocelar; vertical externa subigual às pós-oculares; demais cerdas ocipitais um pouco menores do que as oculares, restritas à metade superior do pós-crânio. Gena com 2-3 cerdas curtas, delgadas; pós-gena com 1 cerda delgada. Probóscide amarela, da altura da cabeça. Antena castanho-escura, exceto pedicelo castanho-claro; flagelo piloso, 3 vezes mais comprido do que o escapo e pedicelo juntos e 4 vezes mais comprido do que o estilo. Tórax: amarelo com pruinosidade cinza, mais conspícua na região posterior do escudo, no escutelo e no subescutelo. Cerdas: 6 aprn distintas, dispostas em leque; 1 pprn forte e 2 fracas; $1-2$ prepm pequenas; aparentemente $3 \mathrm{dc}$ (danificadas pelo alfinete); 1 spal presut; spal psut ausente; $1 \mathrm{npl}$ forte e 1 - 2 fracas; 1 pal; 2 sctl, a interna maior, convergente; 4 ktg. Pernas: amarelas, exceto ápice do fêmur médio e posterior, metade apical da tíbia e todo tarso posterior castanho-escuros, brilhantes; tarsos anterior e médio castanhos. Perna posterior (fig. 1) com fêmur levemente clavado na metade distal, 1 cerda anterior subapical e 3-4 cerdas anteroventrais mais curtas, porém fortes no $1 / 4$ distal; tíbia com 7 cerdas dorsais longas, equidistantes entre si; primeiro tarsômero com série de 4-5 cerdas dorsais. Asa (fig. 10): hialina, com enfuscação castanha nas margens costal e distal atingindo levemente o ápice da célula $r_{4+5}$. Halter castanho. Abdômen: predominantemente castanho-avermelhado com cerdas da margem posterior maiores. Terminália (fig. 2) preta. Tergito 8 (fig. 3) de ápice mais estreito do que a base. Epândrio estreito com cerdas mais fortes dorsalmente; hipândrio alongado; falo agudo distalmente; cerco dividido em dois lóbulos, o anterior maior, o posterior curvo para trás com duas cerdas apicais distintas. 


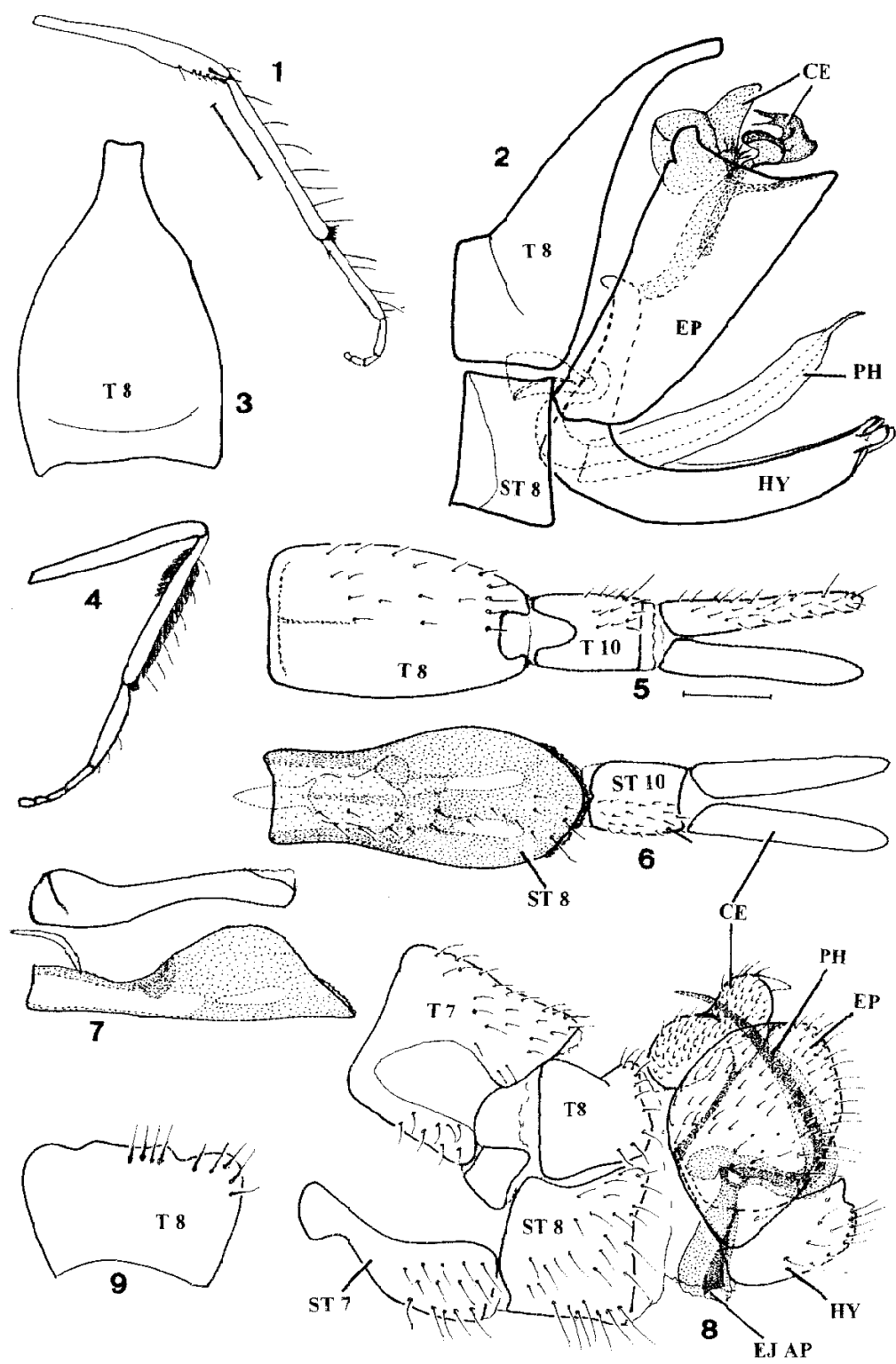

Figs. 1-9. Macrostomus fulvithorax (Curran): 1-3, holótipo o': 1, perna posterior esquerda (exceto coxa e trocanter), vista anterior; 2, terminália, vista lateral esquerda; 3 , tergito 8 , vista dorsal; 4-7, alótipo $9: 4$, perna posterior esquerda (exceto coxa e trocanter), vista anterior; 5 , terminália, vista dorsal; 6 , idem, vista ventral; 7 , idem, segmento 8, vista lateral. Porphyrochroa fasciventris (Curran), holótipo o': 8, terminália, vista lateral esquerda; 9 , tergito 8, vista dorsal. Abreviaturas: CE, cerco; EJ AP, apódema ejaculador; EP, epândrio; HY, hipândrio; PH, falo; ST, esternito; T, tergito. Escalas: 0,5 mm, figs. 1, 4; 0,1 mm, figs. 2, 3, 5-9. 
Fêmea. Corpo 4,2 mm; asa 3,8 mm de comprimento. Semelhante ao macho, exceto nos seguintes caracteres: fronte mais larga do que a face; pós-crânio com área pruinosa menor; tíbia média com 2 cerdas dorsais, sendo uma mediana e uma apical e 1 cerda anteroventral e 1 posteroventral distais; tíbia posterior (fig. 4) castanho-escura a preta, achatada lateralmente, com cerdas peniformes curtas, dorsalmente por toda longitude, exceto no extremo basal e distal, ventralmente com cerdas peniformes mais curtas no 1/ 3 basal; primeiro tarsômero levemente intumescido, com 2-3 cerdas na 1/2 distal, mais curtas do que no macho (fig. 4). Asa (fig. 11) mais larga, com enfuscação mais intensa.

Terminália: tergito 10 com incisão basal (fig. 5); cercos longos, cilíndricos; esternito 8 (fig. 6) com duas manchas claras paramedianas próximo ao ápice; em vista lateral (fig. 7) com metade distal mais larga e ápice serrilhado.

Registro geográfico. Panamá.

Material examinado. PANAMÁ. Canal Zone, Barro Colorado Island, 21.XII.1928, C.H.Curran col. (holótipo ơ", alótipo ९: in copula, AMNH).

Condição do holótipo. Asa esquerda montada em microlâmina; terminália em microtubo com glicerina, ambos afixados no mesmo alfinete do espécime.

\section{Porphyrochroa Melander, 1928, revalidado}

Porphyrochroa Melander, 1928:137 (espécie-tipo Sciodromia palliata Coquillett, 1902, des. orig.).

O gênero Porphyrochroa foi negligenciado ao longo do tempo e foi sinonimizado com Macrostomus por Sмiтн (1967). Os dois são muito próximos entre si mas pertencem a linhagens distintas. Porphyrochroa possui célula dm curta (fig. 12), terminália masculina com hipândrio curto, epândrio suboval e cerco alongado, não dividido (fig. 8). Macrostomus possui célula dm longa (figs. 10,11), hipândrio longo, epândrio estreito, alongado e cerco com dois lobos (fig. 2). As espécies-tipo dos dois gêneros foram examinadas e apresentam, respectivamente, o mesmo padrão das espécies redescritas neste trabalho. As demais espécies atualmente em Macrostomus devem ser revisadas. Macrostomus tem distribuição neotropical, essencialmente amazônica, com poucas espécies circum-amazônicas. Porphyrochroa tem distribuição neotropical, excluindo o Chile, atingindo o sul do Texas nos Estados Unidos.

Material examinado. MÉXICO. Tab[asco], Frontera, 2.19 (sic) [? 02 de fevereiro], Towsend coll., type $\mathrm{n}^{\circ}$. 6315, síntipo $\sigma^{-7}$ de palliata (Coquillett), NMNH. America Meridionalis, Smidt (sic), lectotype $\sigma$ de $M$. ferrugineus (Fabricius, 1805), ZM.

\section{Porphyrochroa fasciventris (Curran, 1931), comb. nov.}

(Figs. 8, 9, 12)

Axelempis fasciventris CURRAN, 1931:10.

Macrostomus fasciventris; SмIтн, 1961:54; 1967:30 (cat.).

Diagnose. Castanho-escura a preta, brilhante, com antena amarela até a base do flagelo, base do halter, probóscide e pernas amarelos; quarto segmento abdominal amarelo-escuro; asa hialina; cerdas torácicas pequenas, exceto uma notopleural forte. 
Holótipo or. Corpo 3,1 mm; asa 2,7 mm de comprimento. Cabeça: olhos dicópticos, castanhos. Fronte preta, brilhante, muito estreita, metade da largura de um ocelo. Face mais larga do que a fronte, da largura de um ocelo, da mesma altura e cor da fronte. Cerdas ocelares pequenas, aparentemente paralelas, proclinadas; um par de cerdas pósocelares diminutas. Pós-crânio estreito, castanho-escuro a preto, brilhante, com esparsa pruinosidade cinza-marrom a certa luz; cerdas pós-oculares unisseriadas, curtas; cerdas vertical interna e externa delgadas, subiguais às ocelares; demais ocipitais restritas à

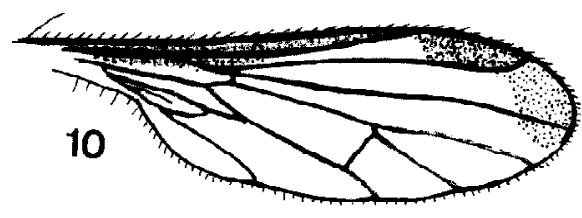

metade superior. Gena e pós-gena com 23 cerdas delgadas cada uma. Probóscide amarela, aproximadamente da altura da cabeça. Antena amarela até a base do flagelo, a partir daí castanho-escura a preta, fosca. Flagelo piloso, três vezes mais longo que escapo e pedicelo juntos e 4 vezes mais longo que o estilo. Tórax: castanho-escuro a preto, brilhante, com

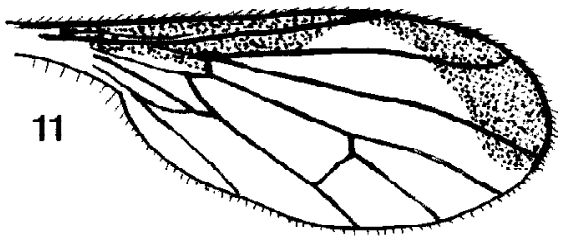
leve pruinosidade cinza, visível a certa luz, na região posterior do escudo, no escutelo e na pleura, esta com uma mancha brilhante mediana, sem pruína, tomando parcialmente o anepisterno, catepisterno, anepímero, catepímero e base do laterotergito. Cerdas (parcialmente danificadas pelo alfinete) pequenas, exceto por uma notopleural mais forte: 1-2 aprn,

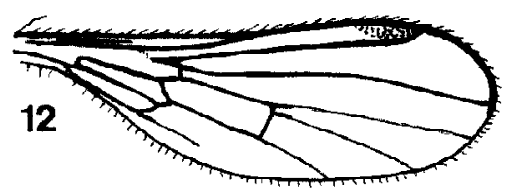

Figs. 10-12. Asas. 10, Macrostomus fulvithorax (Curran), holótipo $\sigma^{-n}$, asa esquerda $(3,9 \mathrm{~mm})$, vista ventral; 11 , idem, alótipo $\uparrow(3,8 \mathrm{~mm}) ; 12$, Porphyrochroa fasciventris (Curran), holótipo o asa direita $(2,7 \mathrm{~mm})$, vista dorsal.

2 pprn, 1 prepm; 8 dc uniseriadas; aparentemente 2 spal presut; spal psut não observada; aparentemente $3 \mathrm{npl}$, sendo uma mais forte; 1 pal; 2 sctl subiguais, ambas convergentes; $2 \mathrm{ktg}$. Pernas: amarelas, exceto tarsos, mais escuros, castanhos a certa luz; pilosidade amarela, curta, sem cerdas fortes que se destaquem das demais. Asa (fig. 12) hialina, com leve enfuscação no ápice da célula $r_{1}$. Halter e tórax concolores, exceto base da haste, amarelo-clara. Abdômen: castanho-escuro a preto, brilhante, exceto o quarto segmento, amarelo-escuro com um largo ponto castanho dorso-apicalmente.

Terminália (fig. 8): tergito 7 com área não esclerotinizada lateralmente e com pequena projeção subtrapezoidal póstero-ventral; tergito 8 mais largo do que seu comprimento (fig. 9). Hipândrio curto; epândrio suboval; cercos com pequena projeção apical; falo longo, afilado distalmente.

Fêmea. Desconhecida.

Registro geográfico. Panamá. 
Material examinado. PANAMÁ. Canal Zone, Barro Colorado Island, 24.XII.1928, C.H.Curran col. (holótipo on, AMNH).

Condição do holótipo. Asa esquerda e pernas médias perdidas; escudo danificado pelo alfinete entomológico. Asa direita montada em microlâmina; terminália em microtubo com glicerina, ambos afixados no mesmo alfinete do espécime.

Agradecimentos. A David Grimaldi, AMNH; Norman Woodley, NMNH e Leif Lyneborg, ZM pelo empréstimo do material.

\section{REFERÊNCIAS BIBLIOGRÁFICAS}

Coquillett, D.W. 1902. New Orthorrhaphous Diptera from Mexico and Texas. JI N. Y. ent. Soc., New York, 10:136-141.

Cumming, J. M.; SinclaiR, B.J. \& Wood, D.M. 1995. Homology and phylogenetic implications of male genitalia in Diptera-Eremoneura. Ent. scand., Copenhagen, 26:120-151.

Curran, C.H. 1931. New species of Empididae from Panama. Am. Mus. Novit., New York, 467:1-12.

McAlpine, J.F. 1981. Morphology and terminology. In: McAlpine, J.F. et al. eds. Manual of Nearctic Diptera. Ottawa, Res. Branch, Agriculture Canada. v.1, p. 9-63. (Monograph nº 27).

Melander, A.L. 1928. Diptera Fam. Empididae. In: Genera Insectorum, Bruxelles, P. Wystman. v. 185,434 p.

Sмiтh, K.G.V. 1961. On the genus Macrostomus Wiedemann (Diptera: Empididae) with notes on the included species and new synonymy. Proc. R. ent. Soc. Lond., (B), London, 30(3-4):53-56.

1967. Family Empididae (Empidae, Hybotidae). In: A catalogue of the Diptera of the Americas South of the United States. São Paulo, Departamento de Zoologia, Secretaria de Agricultura. v. 39, 67 p.

Recebido em 23.03.2000; aceito em 04.09.2000. 